\title{
Radial artery ultrasound predicts the success of transradial coronary angiography
}

\author{
Rami M. Abazid, Osama A. Smettei, Mohamed Z. Mohamed, \\ M. Obadah Kattea, Annie Suresh, Yaser Bashir, Haitham Sakr \\ King Fahad Specialist Hospital, Prince Sultan Cardiac Center Qassim, Buraydah, Saudi Arabia
}

\begin{abstract}
Background: The transradial approach has become the preferred vascular access during conventional coronary angiography (CCA). Hereby, we evaluated the impact of pre-procedural radial artery diameter (RAD), the cross-sectional area (CSA), and the perimeter on vascular complications (VACs).

Methods: We conducted a single-center prospective analysis of 513 patients who underwent CCA. Radial artery ultrasonography was performed before and after CCA to measure the RAD, CSA, and perimeter.

Results: The average RAD, CSA, and perimeter were $2.60 \pm 0.48 \mathrm{~mm}, 6.2 \pm 3.0 \mathrm{~mm}^{2}$, and $8.9 \pm$ $\pm 1.7 \mathrm{~mm}$, respectively. Vascular complications were reported in 56 (11\%) patients. The RAD, CSA, and perimeter were significantly smaller in patients in whom procedure-related VACs were observed than in those with no complications: $2.3 \pm 0.5$ vs. $2.70 \pm 0.54 \mathrm{~mm}(p=0.0001), 4.9 \pm 2.1 \mathrm{vs} .6 .4 \pm$ $\pm 3 \mathrm{~mm}^{2}(p=0.001)$, and $7.6 \pm 2.1$ vs. $9.2 \pm 1.6 \mathrm{~mm}(p=0.0001)$, respectively. Univariate logistic regression showed that RAD, CSA and perimeter can independently predict VACs (OR 0.833, 95\% CI $0.777-0.894, p<0.0001$; OR 0.647, 95\% CI 0.541-0.773, p < 0.0001; OR 0.545, 95\% CI 0.446$-0.665, p<0.0001$, respectively).

Conclusions: Ultrasonographic study of the radial artery before CCA can provide valuable information regarding vascular access. (Cardiol J 2017; 24, 1: 9-14)

Key words: transradial approach, vascular access complications, radial artery diameter, radial artery cross-sectional area, radial artery perimeter
\end{abstract}

\section{Introduction}

Transradial-approach (TRA) coronary angiography, the preferred access for conventional coronary angiography (CCA), results in early ambulation of patients and lower rates of vascular access complications (VACs) and major bleeding [1-7]. With TRA, there is minimal risk of entry site-related complications; around $5 \%$ of patients may have clinical evidence of radial artery occlusion (RAO), which may persist in $2.8 \%$ [8]. In addition, perforation and chronic regional pain syndrome occur only rarely [9, 10]. Radial artery Doppler ultrasound imaging has a major role in investigating post-procedure complications [11]. Radial artery VACs are associated with a smaller radial artery diameter (RAD) [12]; however, there are no data regarding the effect of the radial artery cross-sectional area (CSA) and perimeter. The purpose of the present study was, therefore, to evaluate the impact of pre-procedure RAD, CSA, and perimeter, as determined by ultrasound, on the rate of VACs.

Address for correspondence: Rami Abazid, MD, King Fahad Specialist Hospital, PrinceSultan Cardiac Center Qassim (PSCCQ), King Addullah Street, PO Box: 4560, 51412 Qassim Buraydah, Saudi Arabia, e-mail: ramiabazid@yahoo.com Note: The abstract was accepted for EURO PCR 2015 in Paris as an oral presentation (when the patients' number reached 207 patients). Recently, the abstract has been accepted as highest ranked abstract submitted from Saudi Arabia (received official recognition) during the American College of Cardiology meeting April 2016 Chicago.

Received: 11.06.2016 Accepted: 27.07.2016 


\section{Methods}

\section{Patient seletion}

We performed a single-center prospective study of 513 consecutive patients referred to Prince Sultan Cardiac Center Qassim for CCA. Patients were enrolled between April 2014 and July 2015. Patients referred for primary percutaneous coronary intervention (pPCI) and those with a history of trans-radial CCA were excluded. The study protocol was approved by the regional ethics committee, and all patients provided written informed consent.

\section{Radial artery Doppler ultrasound}

Pre-procedural ultrasound study was performed for all patients using a Vivid $i$ ultrasound system (Vingmed Medical Systems/GE Healthcare, Milwaukee, WI, USA) with a 9L-D linear transducer with a frequency of $13 \mathrm{MHz}$. Pre-procedure cross-sectional imaging of the radial artery was performed by placing the probe $2 \mathrm{~cm}$ proximal to the styloid process of the radius, perpendicular to the vessel wall. Mean RAD was calculated by averaging the maximum and minimum diameters of the radial artery. The perimeter and CSA were measured by tracing the inner vascular lumen (Fig. 1). Next, the probe was aligned longitudinally for color Doppler imaging to assess the presence of blood flow and peak systolic velocity. Image depth and 2-dimensional gain were optimized to obtain the best image quality. In all patients ultrasound assessment of the radial artery was repeated $3-5 \mathrm{~h}$ after the procedure to compare pre- and post-procedure $\mathrm{RAD}, \mathrm{CSA}$, perimeter, and peak systolic velocity and to determine possible complications, such as RAO, perforation, and hematoma.

\section{Coronary angiography protocol}

Conventional coronary angiography was performed using the standard technique. The right radial artery was used for vascular access. After local anesthesia had been achieved with subcutaneous injection of $2 \%$ lidocaine, the radial artery was punctured with a 21-gauge needle. Following this, a 6-French sheath (Terumo Co. Tokyo, Japan) was introduced, and an intra-arterial vasodilator cocktail (verapamil $2 \mathrm{mg}$ and nitroglycerin $200 \mu \mathrm{g}$ ) and 3000 IU of unfractionated heparin were administered. All intra-procedural vascular complications, such as radial artery dissection, radial artery spasm, and conversion to femoral access because of failure of radial arterial access, were recorded. All interventionists in the study had an experience of more than 2 years and had an

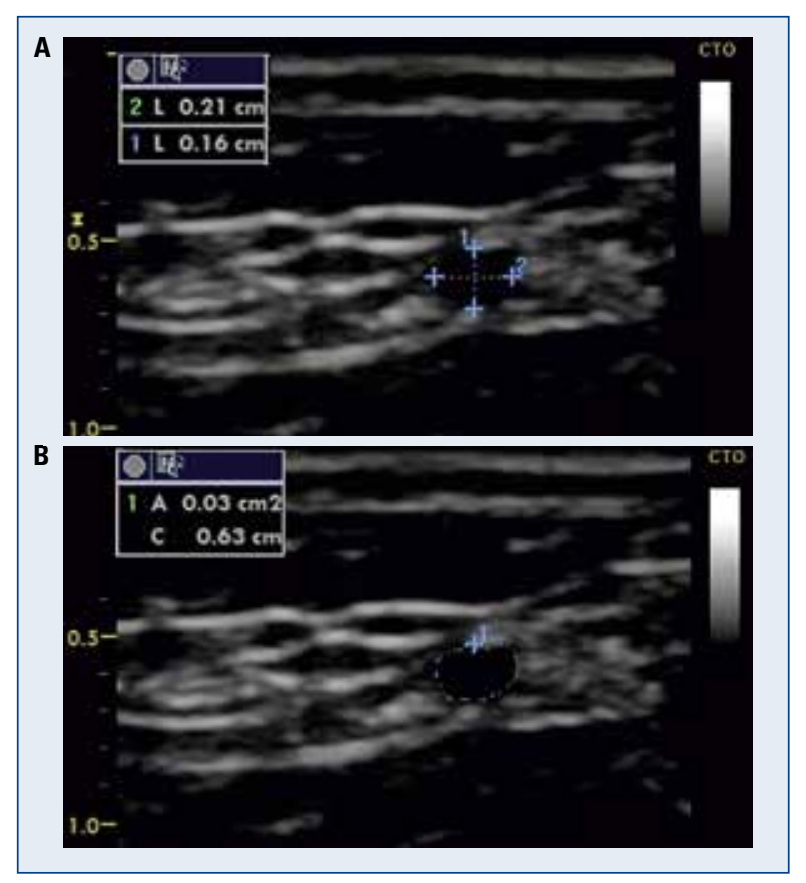

Figure 1. Radial artery ultrasound (RAD); A. Measurement of minimal and maximal diameters of the radial artery; RAD is the average of the two diameters; B. Cross-sectional area and perimeter are calculated by tracing the lumen of the radial artery.

annual rate of more than 75 transradial coronary interventions per year.

\section{Sheath removal and hemostasis methods}

Arterial sheaths were removed immediately after the procedure, then radial compression with a pneumatic device (TR Band of Terumo. Europe, Leuven, Belgium) was placed tightly around the wrist; the band was inflated with $15-18 \mathrm{~mL}$ of air to obtain homeostasis. The pneumatic device was kept in place for $2-3 \mathrm{~h}$ with repeated $2 \mathrm{~mL}$ air withdrawal to reduce inflation pressure. Finally, the tight dressing was applied at puncture site after device removal.

\section{Definition of VACs}

Radial artery occlusion is defined as total obstruction with the absence of flow signal using color flow imaging and pulsed wave Doppler.

Vascular access bleeding is defined as prolong puncture site bleeding despite proper placement of radial compression device or formation of a significant palpable hematoma at the puncture site.

Pseudoaneurysm is defined as arterial wall deficiency, which leads to accumulation of blood in the nearby extra-luminal region with or without clot formation. 


\section{Statistical analysis}

Continuous data are presented as mean \pm standard deviation and categorical variables are expressed as number and percentage. Student's $t$-test was used to analyze the differences between continuous variables and between groups of patients with and without complicated radial artery approach. The $\chi^{2}$ test was used to compare categorical variables. Univariate regression was used to study the association between procedure failure with radial artery ultrasound parameters, and a multivariate logistic regression model determined the ability of the three ultrasound parameters to predict VACs. All statistical analysis was performed using SPSS for Windows, Version 19.0 (SPSS Inc., Chicago, IL, USA). $\mathrm{P}<0.05$ was considered statistically significant for all tests.

\section{Results}

\section{Clinical characteristics}

The mean age was $59 \pm 12$ years. Three hundred sixty-seven $(71.5 \%)$ patients were male. Other clinical characteristics are presented in (Table 1).

\section{Radial ultrasound measurements}

Mean RAD, CSA, and perimeter were $2.6 \pm$ $\pm 0.48 \mathrm{~mm}, 6.2 \pm 3 \mathrm{~mm}^{2}$, and $8.9 \pm 1.7 \mathrm{~mm}$, respectively. Mean RAD, CSA, and perimeter were significantly greater in men than in women $(\mathrm{p}<$ $<0.0001, \mathrm{p}<0.0001$, and $\mathrm{p}=0.0001$, respectively) (Fig. 2).

\section{Coronary artery procedure}

Percutaneous coronary intervention was performed in $354(69 \%)$ patients. Fifty-six (11\%) patients had VACs: $11(2.1 \%)$ had RAO, $12(2.3 \%)$ had vascular-access bleeding, $5(1.2 \%)$ had radial artery dissection, $1(0.2 \%)$ had pseudoaneurysm (Fig. 3 ) and 27 (5.2\%) had severe radial artery spasm requiring conversion to femoral access.

We found that female sex $(\mathrm{p}=0.001)$, smaller $\operatorname{RAD}(\mathrm{p}=0.0001), \operatorname{CSA}(\mathrm{p}=0.001)$, and perimeter $(\mathrm{p}=0.0001)$ were significantly more frequently observed in procedures with complications than in those without complications (Table 2).

\section{Radial ultrasound measurements impact on VACs}

Univariate logistic regression showed that ultrasound measurements can independently predict VACs as follow: odds ratio (OR) 0.833 , $95 \%$ confidence interval (CI) 0.777-0.894, p $<0.0001$ for
Table 1. Baseline clinical characteristics.

\begin{tabular}{lc}
\hline Variables & \\
\hline Age [years] & $59 \pm 12$ \\
Male sex [\%] & $367(71.5 \%)$ \\
Body mass index [kg/m²] & $31.3 \pm 4.2$ \\
Diabetes mellitus [\%] & $308(60 \%)$ \\
Hypertension [\%] & $268(52 \%)$ \\
Dyslipidemia [\%] & $103(20 \%)$ \\
Family history of CAD [\%] & $56(11 \%)$ \\
Current smoking [\%] & $146(28.5 \%)$ \\
Angiographic data & \\
Non-significant CAD [\%] & $82(16 \%)$ \\
Left main artery [\%] & $24(4.5 \%)$ \\
Single vessel [\%] & $193(37.5 \%)$ \\
2 vessels [\%] & $145(28.5 \%)$ \\
3 vessels [\%] & $69(13.5 \%)$ \\
PCl [\%] & $354(69 \%)$ \\
\hline
\end{tabular}

Continuous variables are described as the mean \pm standard deviation and categorical variables as number (proportion); CAD coronary artery disease; $\mathrm{PCI}$ - percutaneous coronary intervention

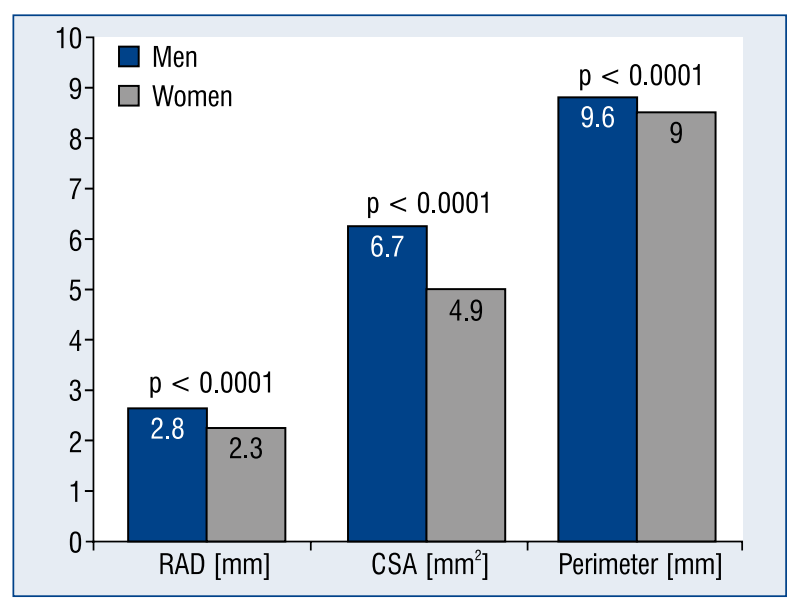

Figure 2. Ultrasound parameters in female and male subsets. Men vs. women had a larger radial diameter, cross-sectional area (CSA), and perimeter; RAD - radial artery diameter.

RDA, OR 0.647, 95\% CI 0.541-0.773, p < 0.0001 for CSA, and OR 0.545, 95\% CI 0.446-0.665, $\mathrm{p}<0.0001$ for perimeter (Table 3 ).

On the other hand, multivariate logistic regression using the radial measurements and other baseline characteristics showed that the radial artery perimeter is the only predictor for VACs as follows: OR 0.49, 95\% CI 0.33-0.726, p $<0.0001$. 


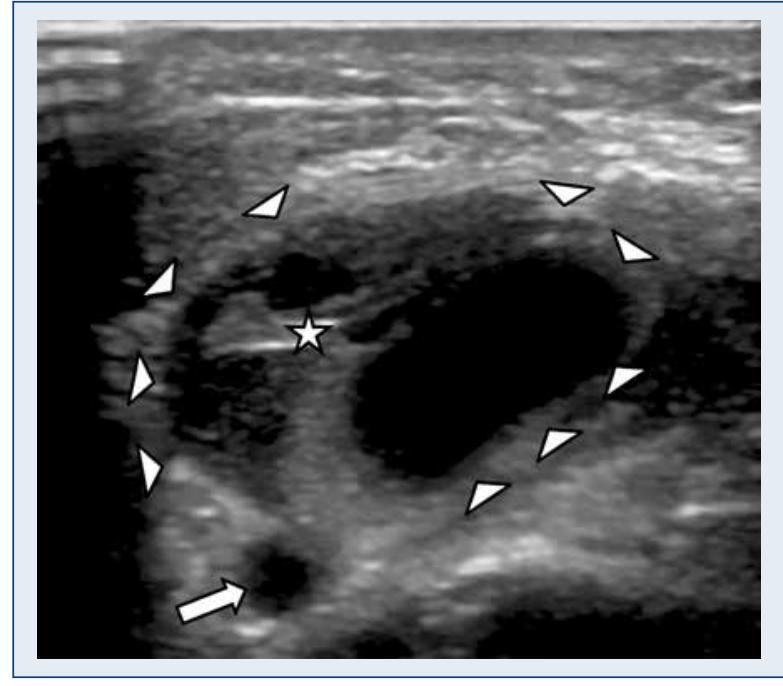

Figure 3. Large radial artery pseudoaneurysm (arrowheads) as a complication after coronary angiography with a partial thrombosis (star); the radial artery indicated by white arrow.

In addition we found that RAD ( $\mathrm{p}=0.021)$, CSA $(p<0.0001)$, and perimeter $(\mathrm{p}<0.0001)$ were significantly smaller pre-procedure than postprocedure (Fig. 4).

Using receiver-operating characteristics curves we found that vascular complications were accurately predicted by ultrasound measurements, with area under the curve (AUC) for RAD: 0.681; 95\% CI 0.595-0.767; $p<0.0001$, AUC for CSA:
Table 3. A. Univariate logistic regression for ultrasound variables and sex shows that radial artery diameter (RAD), cross-sectional area (CSA) and perimeter are strong predictors for vascular access complications (VAC); B. Multivariate logistic regression for ultrasound variables associated with VACs when sex and peak systolic velocity (PSV) is not put in the model, which shows that perimeter is strong predictor for VACs.

\begin{tabular}{|c|c|c|}
\hline Variable & HR $(95 \% \mathrm{CI})$ & $\mathbf{P}$ \\
\hline \multicolumn{3}{|c|}{$\begin{array}{l}\text { A. Univariate logistic regression for variables } \\
\text { associated with VACs }\end{array}$} \\
\hline RAD & $0.833(0.777-0.894)$ & $<0.0001$ \\
\hline CSA & $0.6470 .541-0.773)$ & $<0.0001$ \\
\hline Perimeter & $0.545(0.446-0.665)$ & $<0.0001$ \\
\hline $\begin{array}{l}\text { Peak systolic } \\
\text { velocity }\end{array}$ & $1(0.98-1.02)$ & 0.9 \\
\hline Age & $0.998(0.973-1.022)$ & 0.85 \\
\hline Female sex & $1.3(1.12-1.45)$ & 0.99 \\
\hline \multicolumn{3}{|c|}{$\begin{array}{l}\text { B. Multivariate logistic regression for 2D } \\
\text { ultrasonic variables }\end{array}$} \\
\hline RAD & $0.96(0.84-1.09)$ & 0.5 \\
\hline CSA & $0.99(0.88-1.13)$ & 0.9 \\
\hline Perimeter & $0.49(0.33-0.726)$ & $<0.0001$ \\
\hline
\end{tabular}

$\mathrm{Cl}$ - confidence interval; $\mathrm{HR}$ - hazard ratio

$0.686 ; 95 \%$ CI $0.603-0.770 ; \mathrm{p}<0.0001$, and AUC for perimeter: 0.703 ; $95 \%$ CI $0.621-0.785$; $\mathrm{p}<$ $<0.0001$ (Fig. 5). For the different radial measurements we found that cutoff value of less than

Table 2. The differences between patients with and without vascular access complications (VAC). Patients with VAC had significantly smaller radial artery diameter (RAD), cross-sectional area (CSA) and perimeter.

\begin{tabular}{lccc}
\hline Variables & $\begin{array}{c}\text { Procedure with VACs } \\
(\mathbf{n}=\mathbf{5 6})\end{array}$ & $\begin{array}{c}\text { Successful procedure } \\
(\mathbf{n}=\mathbf{4 5 7 )}\end{array}$ & $\mathbf{P}$ \\
\hline Age [years] & $55 \pm 12$ & $56 \pm 11$ & 0.85 \\
Men [\%] & $28(50 \%)$ & $339(74 \%)$ & 0.001 \\
Women [\%] & $28(50 \%)$ & $118(26 \%)$ & \\
Diabetes mellitus [\%] & $37(66 \%)$ & $271(59 \%)$ & 0.66 \\
Hypertension [\%] & $29(51.8 \%)$ & $239(52 \%)$ & 0.2 \\
Dyslipidemia [\%] & $7(21 \%)$ & $96(10 \%)$ & 0.12 \\
Family history of CAD [\%] & $10(28 \%)$ & $46(10 \%)$ & 0.1 \\
Current smoking [\%] & $9(16 \%)$ & $137(30 \%)$ & 0.079 \\
RAD [mm] & $2.3 \pm 0.5$ & $2.7 \pm 0.54$ & $<0.0001$ \\
Vessel CSA [mm $\left.{ }^{2}\right]$ & $4.9 \pm 2.1$ & $6.4 \pm 3$ & 0.001 \\
Perimeter [mm] & $7.6 \pm 2.1$ & $9.2 \pm 1.6$ & $<0.0001$ \\
Peak systolic velocity [m/s] & $38 \pm 11$ & $37 \pm 15$ & 0.9 \\
\hline
\end{tabular}

Continuous variables are described as the mean \pm standard deviation and categorical variables as number (proportion); CAD — coronary artery disease 


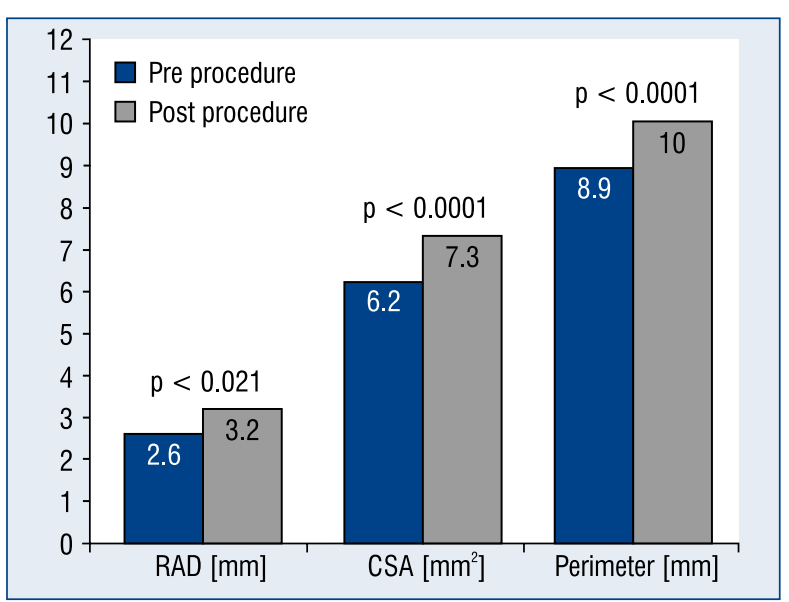

Figure 4. Comparison between pre- and post-procedure ultrasound measurements; showed that the post procedure measures are significantly larger than that of the pre-procedure; RAD — radial artery diameter; CSA - cross-sectional area.

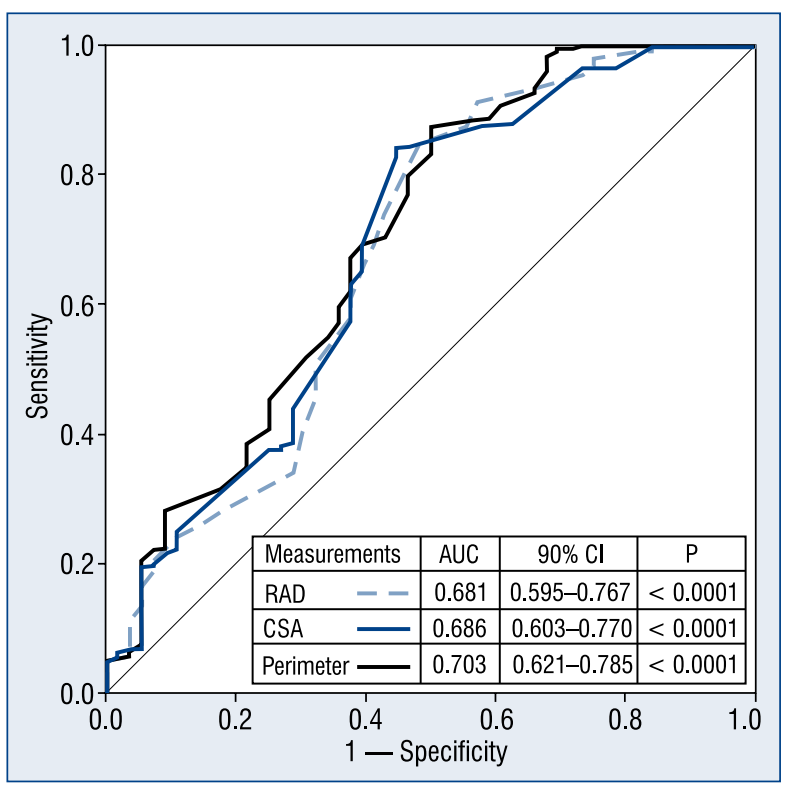

Figure 5. Receiver-operating characteristics curves for radial artery diameter (RAD), cross-sectional area (CSA), and perimeter for detecting vascular access complications.

$2.2 \mathrm{~mm}$ for RDA had 0.69 sensitivity and 0.59 specificity, CSA less than $5 \mathrm{~mm}^{2}$ has 0.68 sensitivity and 0.61 specificity, and perimeter less than $8 \mathrm{~mm}$ has 0.69 sensitivity and 0.61 specificity to predict VACs.

\section{Discussion}

Transradial-approach has become the standard access for CCA because it allows earlier mobility, reduced hospital stay, and decrease rates of VACs $[13,14]$. Furthermore, the indications for TRA have expanded to include the pPCI and treatment of complex lesions, such as those occurring at bifurcations and those creating total occlusion [15-17]. The most frequent complication after TRA is RAO; its incidence of around $5 \%$ may be higher for smaller RADs [8, 12]. Multiple factors, such as sex, wrist circumference, and family history, can predict radial artery size [18]. The Allen test is widely used to evaluate the patency of the radial and ulnar arteries and the sufficiency of the palmar arch collateral circulation before CCA, but the clinical validity of the test before TRA is questionable [19]. Radial artery ultrasound allows measurement of radial diameter and assessment of flow and detection of arterial stenosis, and provides valuable information about vascular anatomy. To our knowledge, this is the first investigation of the role of radial artery CSA and perimeter before TRA.

In the present study, VACs tended to occur with smaller RAD, CSA, and perimeter, and radial artery measurements were significantly larger post-procedure than pre-procedure. Our results are consistent with those of previous reports by Nagai et al. [11] and Yan et al. [12], which showed that smaller radial diameters were associated with higher rates of RAO and procedure failure. In contrast with our findings, Nagai et al. [11] observed significantly smaller post-procedure than pre-procedure RAD. This can be explained by the persistent action of vasodilating medication or the mechanical effect of the arterial sheath dilator caused by earlier ultrasound examination (3-5 h post-procedure) in our study compared with 2 days for Nagai et al. [11].

Radial artery ultrasound is a noninvasive technique that can be performed easily and quickly at bedside. It can also provide important information about vessel anatomy that may predict the procedure's success, mainly in patients expected to have a smaller radial artery lumen such as women or individuals with smaller body habitus. In addition, post-procedure radial ultrasound enables early detection of peri-procedural complications and hence, proper management and follow-up.

\section{Limitations of the study}

Our study had some limitations. First, although $50 \%$ of RAO improves in long-term follow-up, there was no late follow-up ultrasound imaging in our study. Second, only 6-French arterial sheaths were used, which precluded an analysis of the effect of different sheath sizes on the radial artery. Third, 
no patients with transfemoral access were included for comparison with TRA. Forth, patients with the previous transradial coronary procedure were not included in the study. Given the findings, however, the inclusion of these patients would have provided valuable information about the long-term effect of coronary intervention procedure on the radial artery lumen and wall. In addition, the inclusion of these patients would allow the effect of multiple transradial interventions on the incidence rate of VACs to be evaluated, which may guide the selection of the optimal access site for the following procedure. Finally, patients who underwent pPCI were excluded.

\section{Conclusions}

Radial artery ultrasound examination can provide important information before CCA using TRA. In the present study, we found that a smaller radial diameter, CSA, and perimeter were associated with higher rates of VACs.

Funding: This research received no specific grant from any funding agency in the public, commercial or not-for-profit sectors.

\section{Conflict of interest: None declared}

\section{References}

1. Brueck M, Bandorski D, Kramer W, Wieczorek M, Höltgen R, Tillmanns H. A randomized comparison of transradial versus transfemoral approach for coronary angiography and angioplasty. J Am Coll Cardiol Intv, 2009; 2: 1047-1054.

2. Jaffe R, Hong T, Sharieff $\mathrm{W}$ et al. Comparison of radial versus femoral approach for percutaneous coronary interventions in octogenarians. Catheter Cardiovasc Interv, 2007; 69: 815-820.

3. Jolly SS, Amlani S, Hamon M et al. Radial versus femoral access for coronary angiography or intervention and the impact on major bleeding and ischemic events: A systematic review and metaanalysis of randomized trials. Am Heart J, 2009; 157: 132-140.

4. Louvard Y, Benamer H, Garot P et al. Comparison of transradial and transfemoral approaches for coronary angiography and angioplasty in octogenarians (the OCTOPLUS study). Am J Cardiol, 2004; 94: 1177-1180.

5. Pristipino C, Trani C, Nazzaro MS et al. Major improvement of percutaneous cardiovascular procedure outcomes with radial artery catheterisation: Results from the PREVAIL study. Heart, 2009; 95: 476-482.
6. Rao SV, Ou FS, Wang TY et al. Trends in the prevalence and outcomes of radial and femoral approaches to percutaneous coronary intervention: A report from the National Cardiovascular Data Registry. J Am Coll Cardiol Intv, 2008; 1: 379-386.

7. Rao SV, Cohen MG, Kandzari DE et al. The transradial approach to percutaneous coronary intervention: Historical perspective, current concepts, and future directions. J Am Coll Cardiol, 2010; 55: 2187-2195.

8. Stella PR, Kiemeneij F, Laarman GJ et al. Incidence and outcome of radial artery occlusion following transradial artery coronary angioplasty. Cathet Cardiovasc Diagn, 1997; 40: 156-158.

9. Calviño-Santos RA, Vázquez-Rodríguez JM, Salgado-Fernández J et al. Management of iatrogenic radial artery perforation. Catheter Cardiovasc Interv, 2004; 61: 74-78.

10. Papadimos TJ, Hofmann JP. Radial artery thrombosis, palmar arch systolic blood velocities, and chronic regional pain syndrome 1 following transradial cardiac catheterization. Catheter Cardiovasc Interv, 2002; 57: 537-540.

11. Nagai S, Abe S, Sato T, et al. Ultrasonic assessment of vascular complications in coronary angiography and angioplasty after transradial approach. Am J Cardiol, 1999; 83: 180-186.

12. Yan ZX, Zhou YJ, Zhao YX, Zhou ZM, Yang SW, Wang ZJ. Anatomical study of forearm arteries with ultrasound for percutaneous coronary procedures. Circ J, 2010; 74: 686-692.

13. Cooper CJ, El-Shiekh RA, Cohen DJ et al. Effect of transradial access on quality of life and cost of cardiac catheterization: A randomized comparison. Am Heart J, 1999; 138: 430$-436$.

14. Vorobcsuk A, Kónyi A, Aradi D et al. Transradial versus transfemoral percutaneous coronary intervention in acute myocardial infarction: Systematic overview and meta-analysis. Am Heart J, 2009; 158: 814-821.

15. Kim JY, Yoon J, Jung HS et al. Feasibility of the radial artery as a vascular access route in performing primary percutaneous coronary intervention. Yonsei Med J, 2005; 46: 503-510.

16. Saito S, Tanaka S, Hiroe $Y$ et al. Comparative study on transradial approach vs. transfe-moral approach in primary stent implantation for patients with acute myocardial infarction: Results of the test for myocardial infarction by prospective unicenter randomization for access sites (TEMPURA) trial. Catheter Cardiovasc Interv, 2003; 59: 26-33.

17. Yang YJ, Xu B, Chen JL et al. Comparison of immediate and follow up results between transradial and transfemoral approach for percutaneous coronary intervention in true bifurcational lesions. Chin Med J, 2007; 120: 539-544.

18. Kotowycz MA, Johnston KW, Ivanov J et al. Predictors of radial artery size in patients undergoing cardiac catheterization: Insights from the Good Radial Artery Size Prediction (GRASP) study. Can J Cardiol, 2014; 30: 211-216.

19. Valgimigli M, Campo G, Penzo $\mathrm{C}$ et al. Transradial coronary catheterization and intervention across the whole spectrum of Allen test results. J Am Coll Cardiol, 2014; 63: 1833-1841. 